\title{
Asymptotic dynamics in 3D gravity with torsion
}

\author{
M. Blagojević and M. Vasilić* \\ Institute of Physics, P.O.Box 57, 11001 Belgrade, Serbia
}

\begin{abstract}
We study the nature of boundary dynamics in the teleparallel 3D gravity. The asymptotic field equations with anti-de Sitter boundary conditions yield only two non-trivial boundary modes, related to a conformal field theory with classical central charge. After showing that the teleparallel gravity can be formulated as a Chern-Simons theory, we identify dynamical structure at the boundary as the Liouville theory.
\end{abstract}

\section{INTRODUCTION}

The growth of interest in three-dimensional (3D) gravity in the past twenty years is motivated by the hope that such a simple model might help us to get a better understanding of the intricate structure of the realistic four-dimensional theory $[1,2]$. Particularly important advances along these lines were achieved by clarifying the asymptotic structure of 3D gravity [3], and formulating 3D gravity as a Chern-Simons theory [4]. These results have a significant influence on our understanding of the quantum nature of 3D black holes [5-12]. However, the analysis of these issues has been carried out almost exclusively in the realm of Riemannian geometry of general relativity (GR). The investigation of the asymptotic structure of 3D gravity in the context of Riemann-Cartan geometry, a geometry possessing both the curvature and the torsion [13,14], has been initiated in our resent publication [15].

The asymptotic content of gravity is most clearly seen in topological theories, where the non-trivial dynamics can exist only at the boundary. Mielke and Baekler [16,17] proposed a general action for topological Riemann-Cartan 3D gravity. The analysis of its classical solutions has been recently completed in Ref. [18]. For a specific choice of free parameters, this theory leads to the teleparallel (or Weitzenböck) geometry of spacetime [19-21,14], in which curvature vanishes and torsion remains non-trivial. This limit is particularly simple framework for studying the influence of torsion on the boundary dynamics. After having clarified the nature of asymptotic symmetry in the teleparallel 3D gravity [15], we now continue with the analysis of the related asymptotic dynamics.

Riemann-Cartan theory can be formulated as Poincaré gauge theory [13,14]. Basic gravitational variables are the triad field $b^{i}{ }_{\mu}$ and the Lorentz connection $A^{i j}{ }_{\mu}$, and the related field strengths are the torsion $T^{i}{ }_{\mu \nu}$ and the curvature $R^{i j}{ }_{\mu \nu}$. In 3D, we can simplify the

*Email addresses: mb@phy.bg.ac.yu,mvasilic@phy.bg.ac.yu 
notation by introducing

$$
\omega_{i \mu}=-\frac{1}{2} \varepsilon_{i j k} A_{\mu}^{j k}, \quad R_{i \mu \nu}=-\frac{1}{2} \varepsilon_{i j k} R_{\mu \nu}^{j k} .
$$

Then, the gauge transformations of the fields, local Lorentz transformations and local translations with parameters $\theta^{i}$ and $\xi^{\mu}$, take the form

$$
\begin{aligned}
\delta_{0} b^{i}{ }_{\mu} & =\varepsilon^{i j k} \theta_{j} b_{k \mu}-\xi^{\rho}{ }_{, \mu} b^{i}{ }_{\rho}-\xi^{\rho} \partial_{\rho} b^{i}{ }_{\mu} \\
\delta_{0} \omega^{i}{ }_{\mu} & =-\nabla_{\mu} \theta^{i}-\xi^{\rho}{ }_{, \mu} \omega^{i}{ }_{\rho}-\xi^{\rho} \partial_{\rho} \omega^{i}{ }_{\mu},
\end{aligned}
$$

where $\nabla_{\mu} \theta^{i}=\partial_{\mu} \theta^{i}+\varepsilon_{m n}^{i} \omega^{m}{ }_{\mu} \theta^{n}$, and the field strengths are given as

$$
\begin{aligned}
& T_{\mu \nu}^{i}=\partial_{\mu} b^{i}{ }_{\nu}+\varepsilon^{i j k} \omega_{j \mu} b_{k \nu}-(\mu \leftrightarrow \nu), \\
& R_{\mu \nu}^{i}=\partial_{\mu} \omega^{i}{ }_{\nu}-\partial_{\nu} \omega^{i}{ }_{\mu}+\varepsilon^{i j k} \omega_{j \mu} \omega_{k \nu} .
\end{aligned}
$$

Starting from the general Mielke-Baekler action [16,17], the gravitational sector of our Riemann-Cartan theory is assumed to have a specific form, suitable for studying the role of torsion at the boundary [15]:

$$
\begin{aligned}
I_{G} & =\int_{\mathcal{M}} d^{3} \xi \mathcal{L}_{G}, \\
\mathcal{L}_{G} & =a\left[\varepsilon^{\rho \mu \nu} b_{\rho}^{i}\left(R_{i \mu \nu}-\frac{1}{\ell} T_{i \mu \nu}\right)+\frac{8}{\ell^{2}} b\right] .
\end{aligned}
$$

Here, $a=1 / 16 \pi G$, $\ell$ is a constant, $b=\operatorname{det}\left(b^{i}{ }_{\mu}\right)$, and $\mathcal{M}$ is a manifold with the topology $R \times \Sigma$, where $R$ is interpreted as time, and $\Sigma$ is a spatial manifold with the asymptotic region of $R^{2}$ type. The related field equations in vacuum,

$$
R_{\mu \nu}^{i}=0, \quad T_{\mu \nu}^{i}=\frac{2}{\ell} \varepsilon^{i}{ }_{j k} b^{j}{ }_{\mu} b^{k}{ }_{\nu},
$$

imply that the geometry of spacetime at large distances, outside the regions occupied by matter, is teleparallel.

In addition to the field equations, asymptotic conditions play an equally important role in defining the dynamical structure of a field theory. Our study of the asymptotic symmetry of the teleparallel theory (1.2) is based on the concept of an asymptotically anti-de Sitter (AdS) configuration of fields, which is precisely defined by the requirements (4.2) in Ref. [15]. The canonical realization of the asymptotic symmetry is found to be the same as in GR - the conformal symmetry with classical central charge $c=3 \ell / 2 G$. The subject of the present paper is to precisely identify the related asymptotic dynamics, whereupon we shall be able to completely understand the dynamical role of torsion at the boundary of the teleparallel spacetime.

The paper is organized as follows. In Sec. II we study the dynamical content of the gravitational field equations in the asymptotic (boundary) region, and show that it can be described by two independent boundary modes - a left moving and a right moving mode. In Sec. II we find a simple change of variables, $\left(b^{i}{ }_{\mu}, \omega^{i}{ }_{\mu}\right) \rightarrow\left(A^{i}{ }_{\mu}, \bar{A}^{i}{ }_{\mu}\right)$, which transforms the teleparallel theory (1.2) into a Chern-Simons theory, in analogy with Witten's result for GR [4]. In Sec. IV, using the new variables we establish the equivalence of the teleparallel 
boundary dynamics with Liouville theory, again in analogy with GR [5]. These results lead us to an important conclusion: in spite of different geometric content, the boundary dynamics in the teleparallel theory is exactly the same as in GR. Finally, Sec. V is devoted to concluding remarks, and appendices contain some technical details.

Our conventions are the same as in Ref. [15]: the Latin indices $(i, j, k, \ldots)$ refer to the local Lorentz frame, the Greek indices $(\mu, \nu, \rho, \ldots)$ refer to the coordinate frame, and both run over $0,1,2 ; \eta_{i j}=(+,-,-)$ and $g_{\mu \nu}=b^{i}{ }_{\mu} b^{j}{ }_{\nu} \eta_{i j}$ are metric components in the local Lorentz and coordinate frame, respectively; totally antisymmetric tensor $\varepsilon^{i j k}$ and the related tensor density $\varepsilon^{\mu \nu \rho}$ are both normalized by $\varepsilon^{012}=+1$.

\section{BOUNDARY DYNAMICS 1}

In Riemann-Cartan spacetime, we can use a well known geometric identity to express the curvature tensor in terms of its Riemannian piece $\tilde{R}_{\mu \nu \lambda \rho}$ and the contortion $[14,15]$. Then, the field equations (1.3) can be rewritten as

$$
\tilde{R}_{\mu \nu \lambda \rho}=\frac{1}{\ell^{2}}\left(g_{\mu \lambda} g_{\nu \rho}-g_{\mu \rho} g_{\nu \lambda}\right), \quad T_{\mu \nu}^{i}=\frac{2}{\ell} \varepsilon^{i}{ }_{j k} b_{\mu}^{j} b_{\nu}^{k} .
$$

The torsion equation eliminates the connection $\omega^{i}{ }_{\mu}$ as an independent dynamical variable, and we are left with the maximally symmetric metric $g_{\mu \nu}$, corresponding to the AdS space [22]. As maximally symmetric spaces are locally unique, we conclude that our theory carries no local degrees of freedom. Instead, all the solutions of the theory are gauge equivalent, at least in local regions of spacetime.

Although the total number of local degrees of freedom is zero, the theory turns out to have a rich asymptotic structure. Namely, after adopting a suitable set of asymptotic conditions (defined by the AdS asymptotic configurations of fields), one finds that the complete gauge symmetry of the theory can be naturally split up into two parts: a) the asymptotic symmetry and b) the remaining proper gauge symmetry $[15,23,3]$. The first acts on the boundary, has the structure isomorphic to the two-dimensional conformal group, and is produced by the generators with non-vanishing conserved charges. The second acts on the spacetime interior, and has generators with vanishing conserved charges.

To a great extent, the physical (geometric) interpretation of the above symmetry structure is motivated by the related quantum theory of gravity [2,5-11]. At the quantum level, the asymptotic symmetry is broken (conformal anomaly), and therefore, it should not be treated as a genuine gauge symmetry, but as a physical one. At the same time, the proper gauge symmetry remains truly non-physical. The existence of the proper gauge freedom implies that the whole interior of spacetime is pure gauge, and carries no local dynamical degrees of freedom. These are located only on the boundary of the underlying spacetime. Classical theory with such an interpretation of its symmetry structure has a direct relevance for the corresponding quantum theory (see also Refs. [24]).

The subject of the present paper is to identify the boundary degrees of freedom associated with the breakdown of gauge invariance at the boundary. Their number is expected to be the same as the number of parameters of the conformal symmetry. Aware of the fact that the spacetime interior carries no local degrees of freedom, we shall focus our attention solely on the dynamical properties at the boundary. 
The asymptotic configuration of the basic dynamical variables is defined using the concept of the AdS asymptotic behavior [3,23]. It is given by the expressions [15]

$$
\begin{gathered}
b_{\mu}^{i}=\left(\begin{array}{ccc}
\frac{r}{\ell}+\frac{\ell}{r} \hat{B}_{0}^{0} & \frac{\ell^{4}}{r^{4}} \hat{B}^{0}{ }_{1} & \frac{\ell}{r} \hat{B}^{0}{ }_{2} \\
\frac{\ell^{2}}{r^{2}} \hat{B}_{0}{ }_{0} & \frac{\ell}{r}+\frac{\ell^{3}}{r^{3}} \hat{B}^{1}{ }_{1} & \frac{\ell^{2}}{r^{2}} \hat{B}^{1}{ }_{2} \\
\frac{\ell}{r} \hat{B}^{2}{ }_{0} & \frac{\ell^{4}}{r^{4}} \hat{B}^{2}{ }_{1} & r+\frac{\ell}{r} \hat{B}^{2}{ }_{2}
\end{array}\right), \\
\omega^{i}{ }_{\mu}=\left(\begin{array}{ccc}
\frac{r}{\ell^{2}}+\frac{\ell}{r} \hat{\Omega}_{0}^{0} & \frac{\ell^{4}}{r^{4}} \hat{\Omega}^{0}{ }_{1} & -\frac{r}{\ell}+\frac{\ell}{r} \hat{\Omega}^{0}{ }_{2} \\
\frac{\ell^{2}}{r^{2}} \hat{\Omega}^{1}{ }_{0} & \frac{1}{r}+\frac{\ell^{3}}{r^{3}} \hat{\Omega}^{1}{ }_{1} & \frac{\ell^{2}}{r^{2}} \hat{\Omega}^{1}{ }_{2} \\
-\frac{r}{\ell^{2}}+\frac{\ell}{r} \hat{\Omega}^{2}{ }_{0} & \frac{\ell^{4}}{r^{4}} \hat{\Omega}^{2}{ }_{1} & \frac{r}{\ell}+\frac{\ell}{r} \hat{\Omega}^{2}{ }_{2}
\end{array}\right),
\end{gathered}
$$

where $x^{\mu}=(t, r, \varphi)$. In Ref. [15], the variables $\omega^{0}{ }_{1}$ and $\omega^{2}{ }_{1}$ behaved as $1 / r^{2}$ at spatial infinity. However, solving the constraints of the theory in the asymptotic region, one discovers $\omega^{0}{ }_{1}, \omega^{2}{ }_{1}=\mathcal{O}_{4}\left(\mathcal{O}_{n}\right.$ denotes a quantity that tends to zero as $1 / r^{n}$ or faster when $\left.r \rightarrow \infty\right)$. We decided to take this result into account from the very beginning. Particularly important solution of the type (2.1) is the black hole solution [25].

The new variables $\hat{B}^{i}{ }_{\mu}$ and $\hat{\Omega}^{i}{ }_{\mu}$ are defined to be of the $\mathcal{O}_{0}$ type. In what follows, we shall be interested only in the lowest order terms (zero modes) of $\hat{B}, \hat{\Omega}$, whereas the higher order terms are expected to be pure gauge terms, hence physically irrelevant. As we shall see, the forthcoming results will justify this approach.

The adopted boundary conditions (2.1) restrict the gauge parameters $\xi^{\mu}, \theta^{i}$ to have the form $[15]$

$$
\begin{aligned}
\xi^{0} & =\ell T+\frac{\ell^{5}}{2 r^{2}} T_{, 00}+\frac{\ell^{4}}{r^{4}} \hat{\xi}^{0}, & \theta^{0} & =-\frac{\ell^{2}}{r} T_{, 02}+\frac{\ell^{3}}{r^{3}} \hat{\theta}^{0}, \\
\xi^{1} & =-r \ell T_{, 0}+\frac{\ell}{r} \hat{\xi}^{1}, & \theta^{1} & =T_{, 2}+\frac{\ell^{2}}{r^{2}} \hat{\theta}^{1}, \\
\xi^{2} & =S-\frac{\ell^{2}}{2 r^{2}} S_{, 22}+\frac{\ell^{4}}{r^{4}} \hat{\xi}^{2}, & \theta^{2} & =\frac{\ell^{3}}{r} T_{, 00}+\frac{\ell^{3}}{r^{3}} \hat{\theta}^{2}
\end{aligned}
$$

The parameters $T$ and $S$ satisfy the equations $\partial_{ \pm}(T \mp S)=0$ and define the asymptotic symmetry group of the theory (the conformal group with classical central charge $3 \ell / 2 G$ ). The proper gauge transformations are defined by the remaining parameters $\hat{\xi}^{\mu}$, $\hat{\theta}^{i}$, which are arbitrary functions of the $\mathcal{O}_{0}$ type. In what follows, the proper gauge parameters $\hat{\xi}^{\mu}, \hat{\theta}^{i}$ will be used to remove the unphysical modes at the boundary.

To simplify the notation, we utilize the light-cone basis to define a) the coordinate and b) local Lorentz light-cone components of vectors:

$$
\begin{aligned}
V^{\mu}: & V^{ \pm}=\frac{1}{\ell} V^{0} \pm V^{2}, & V_{ \pm}=\frac{1}{2}\left(\ell V_{0} \pm V_{2}\right), \\
U^{i}: & U^{ \pm}=U^{0} \pm U^{2}, & U_{ \pm}=\frac{1}{2}\left(U_{0} \pm U_{2}\right) .
\end{aligned}
$$


Note that with these conventions, the light-cone coordinates are dimensionless: $x^{ \pm}=t / \ell \pm \varphi$.

The action of the proper gauge group on $\hat{B}$ and $\hat{\Omega}$ can be found from the general transformation law (1.1a). It is given in Appendix A, and can be used to fix the proper gauge freedom in the lowest order. In particular, we can choose the following six gauge conditions:

$$
\begin{array}{ll}
\hat{B}_{1}^{ \pm}=\mathcal{O}_{1}, & \hat{B}_{1}^{1}=\mathcal{O}_{1}, \\
\hat{\Omega}_{1}^{ \pm}=\mathcal{O}_{1}, & \hat{\Omega}_{1}^{1}=\mathcal{O}_{1} .
\end{array}
$$

After fixing the lowest order terms of $\hat{B}_{1}^{i}$ and $\hat{\Omega}_{1}^{i}$, we are left with $18-6=12$ variables subject to the equations of motion. As we already explained, only the lowest order terms of our variables $\hat{B}, \hat{\Omega}$ can contribute to the boundary dynamics. This means that only those field equations that contain zero modes of the remaining variables are of interest to us. There are exactly 12 such equations. Two of them stem from the constraints of the theory, and they read:

$$
\begin{aligned}
& \hat{\Omega}_{+}^{-}-\hat{\Omega}_{-}^{-}=\mathcal{O}_{1} \\
& 2 \hat{B}_{-}^{+}-\ell \hat{\Omega}_{-}^{+}=2 \hat{B}_{+}^{+}-\ell \hat{\Omega}_{+}^{+}+\mathcal{O}_{1} .
\end{aligned}
$$

The remaining 10 needed equations fall into two categories: 8 equations become asymptotic constraints, as their time derivatives vanish at spatial infinity,

$$
\begin{aligned}
& \hat{B}_{ \pm}^{1}=\mathcal{O}_{1}, \quad \hat{B}_{+}^{+}=\mathcal{O}_{1}, \quad \hat{B}_{-}^{-}=\mathcal{O}_{1}, \\
& \hat{\Omega}_{ \pm}^{1}=\mathcal{O}_{1}, \quad \hat{\Omega}_{+}^{+}=\mathcal{O}_{2}, \quad \hat{\Omega}_{+}^{-}=\mathcal{O}_{2},
\end{aligned}
$$

while the remaining two are proper dynamical equations:

$$
\partial_{+} \hat{B}_{-}^{+}=\mathcal{O}_{1}, \quad \partial_{-} \hat{B}_{+}^{-}=\mathcal{O}_{1} .
$$

As we can see, only the variables $\hat{B}_{-}^{+}$and $\hat{B}_{+}^{-}$have independent non-vanishing zero modes, which means that the boundary dynamics is essentially described by equations (2.5c). The corresponding 2-dimensional theory carries one field degree of freedom. Note that the variables $\hat{B}_{-}^{+}$and $\hat{B}_{+}^{-}$are invariant under the action of the proper gauge transformations on the boundary (Appendix A). Therefore, the form of the equations $(2.5 \mathrm{c})$ is independent of the particular gauge used.

The physical meaning of the fields $\hat{B}_{+}^{-}$and $\hat{B}_{-}^{+}$is seen by the comparison with the energy and angular momentum expressions, defined as the $r \rightarrow \infty$ limit of the following boundary integrals [15]:

$$
E=\int_{0}^{2 \pi} \mathcal{E}^{1} d \varphi, \quad M=\int_{0}^{2 \pi} \mathcal{M}^{1} d \varphi
$$

where

$$
\begin{aligned}
\mathcal{E}^{1} & =2 a\left(\omega^{0}{ }_{2}+\frac{1}{\ell} b^{2}{ }_{2}-\frac{1}{\ell} b^{0}{ }_{2}\right) b^{0}{ }_{0}, \\
\mathcal{M}^{1} & =-2 a\left(\omega^{2}{ }_{2}+\frac{1}{\ell} b^{0}{ }_{2}-\frac{1}{\ell} b^{2}{ }_{2}\right) b^{2}{ }_{2} .
\end{aligned}
$$

Using the asymptotic field equations (2.5), one finds 


$$
\begin{aligned}
& \mathcal{E}_{+} \equiv \frac{1}{2}\left(\ell \mathcal{E}^{1}+\mathcal{M}^{1}\right)=-2 a \hat{B}_{+}^{-}+\mathcal{O}_{1} \\
& \mathcal{E}_{-} \equiv \frac{1}{2}\left(\ell \mathcal{E}^{1}-\mathcal{M}^{1}\right)=-2 a \hat{B}_{-}^{+}+\mathcal{O}_{1}
\end{aligned}
$$

Thus,

the variables $\hat{B}_{+}^{-}$and $\hat{B}_{-}^{+}$are proportional to the boundary values of the components $\mathcal{E}_{+}$and $\mathcal{E}_{-}$of the energy/angular momentum densities.

Rewritten in terms of $\mathcal{E}_{ \pm}$, the boundary field equations $(2.5 \mathrm{c})$ read: $\partial_{ \pm} \mathcal{E}_{\mp}=\mathcal{O}_{1}$. The densities $\mathcal{E}_{ \pm}$are invariants of the proper gauge group, but transform non-trivially under the conformal transformations [15]:

$$
\begin{aligned}
& \delta_{0} \mathcal{E}_{+}=-T^{+} \partial_{+} \mathcal{E}_{+}-2 \partial_{+} T^{+} \mathcal{E}_{+}+a \ell \partial_{+}^{3} T^{+}+\mathcal{O}_{1}, \\
& \delta_{0} \mathcal{E}_{-}=-T^{-} \partial_{-} \mathcal{E}_{-}-2 \partial_{-} T^{-} \mathcal{E}_{-}+a \ell \partial_{-}^{3} T^{-}+\mathcal{O}_{1},
\end{aligned}
$$

where $T^{ \pm} \equiv T \pm S$. These relations are equivalent to equations (6.19) in Ref. [15], which tells us that the boundary dynamics is described as the conformal field theory with classical central charge $c=24 \pi a \ell=3 \ell / 2 G$. In section IV, we shall explicitly establish the equivalence of our boundary dynamics with the Liouville theory.

\section{CHERN-SIMONS FORMULATION OF THE TELEPARALLEL 3D GRAVITY}

A particularly interesting feature of Riemannian 3D gravity is the fact that it is equivalent to an ordinary gauge theory with a specific interaction of the Chern-Simons type [3]. This result has been used in Ref. [4] to show that the related boundary dynamics coincides with the Liouville theory. In this section, we shall prove that the teleparallel gravity can also be represented as a Chern-Simons theory.

The canonical analysis of the teleparallel theory (1.2) yields the following expressions for its gauge symmetries [15]:

$$
\begin{aligned}
& \delta_{0} b_{\mu}^{i}=\nabla_{\mu} \varepsilon^{i}-\frac{2}{\ell} \varepsilon^{i}{ }_{j k} b_{\mu}^{j} \varepsilon^{k}+\varepsilon^{i}{ }_{j k} b_{\mu}^{j} \tau^{k}, \\
& \delta_{0} \omega^{i}{ }_{\mu}=\nabla_{\mu} \tau^{i} .
\end{aligned}
$$

Introducing the new parameters $\xi^{\mu}$ and $\theta^{i}$ by $\varepsilon^{i}=-\xi^{\mu} b_{\mu}^{i}, \tau^{i}=-\left(\theta^{i}+\xi^{\mu} \omega^{i}{ }_{\mu}\right)$, one finds that these gauge transformations are on-shell equivalent to the gauge transformations (1.1a). Witten's idea that the action of 3D gravity is a combination of two pieces, each of which depends on an independent gauge field, can be tested at the level of gauge transformations, where we expect these two gauge fields to transform independently of each other. After introducing the new variables

$$
A_{\mu}^{i}=\omega_{\mu}^{i}, \quad \bar{A}_{\mu}^{i}=\omega_{\mu}^{i}-\frac{2}{\ell} b_{\mu}^{i}
$$

the gauge transformations (3.1) can be rewritten as 


$$
\begin{array}{rlrl}
\delta_{0} A^{i}{ }_{\mu} & =\partial_{\mu} u^{i}+\varepsilon^{i}{ }_{j k} A^{j}{ }_{\mu} u^{k}, & & u^{i} \equiv \tau^{i}, \\
\delta_{0} \bar{A}_{\mu}^{i}=\partial_{\mu} \bar{u}^{i}+\varepsilon^{i}{ }_{j k} \bar{A}^{j}{ }_{\mu} \bar{u}^{k}, & & \bar{u}^{i} \equiv \tau^{i}-\frac{2}{\ell} \varepsilon^{i} .
\end{array}
$$

The fields $A$ and $\bar{A}$ are recognized as the gauge fields of two independent $S L(2, R)$ gauge groups. Indeed, the fundamental matrix representation of the $S L(2, R)$ generators (real, traceless, $2 \times 2$ matrices) can be chosen as $T_{0}=\frac{1}{2} i \sigma_{2}, T_{1}=\frac{1}{2} \sigma_{3}, T_{2}=\frac{1}{2} \sigma_{1}\left(\sigma_{i}\right.$ are the Pauli matrices), i.e.

$$
T_{0}=\frac{1}{2}\left(\begin{array}{cc}
0 & 1 \\
-1 & 0
\end{array}\right), \quad T_{2}=\frac{1}{2}\left(\begin{array}{ll}
0 & 1 \\
1 & 0
\end{array}\right), \quad T_{1}=\frac{1}{2}\left(\begin{array}{cc}
1 & 0 \\
0 & -1
\end{array}\right) .
$$

The Cartan metric has the form $\gamma_{i j}=-2 \operatorname{Tr}\left(T_{i} T_{j}\right)=\eta_{i j}=(+1,-1,-1)$, and the Lie algebra is given as $\left[T_{i}, T_{j}\right]=\varepsilon_{i j}{ }^{k} T_{k}$, with $\varepsilon_{012}=1$. Thus, the original gauge symmetry (3.1) can be equivalently represented by a set of two independent $S L(2, R)$ gauge symmetries.

Using the variables (3.2a), we can rewrite the original field equations (1.3) in the form

$$
F_{\mu \nu}^{i}(A)=0, \quad F^{i}{ }_{\mu \nu}(\bar{A})=0,
$$

where $F^{i}{ }_{\mu \nu}(A)=\partial_{\mu} A^{i}{ }_{\nu}-\partial_{\nu} A^{i}{ }_{\mu}+\varepsilon^{i}{ }_{j k} A^{j}{ }_{\mu} A^{k}{ }_{\nu}$ is the $S L(2, R)$ gauge field strength.

The existence of two gauge fields with independent transformation laws (3.2b) suggests that the gravitational Lagrangian, when expressed in terms of the new variables (3.2a), consists of two pieces, each of which is a functions of only $A$ or $\bar{A}$. To verify this idea, we perform the change of variables (3.2a) in the gravitational Lagrangian (1.2), and obtain the important identity

$$
\mathcal{L}_{G}+\partial_{\mu} D^{\mu}=\mathcal{L}_{\mathrm{CS}}(A)-\mathcal{L}_{\mathrm{CS}}(\bar{A})
$$

where $\mathcal{L}_{\mathrm{CS}}$ is the Chern-Simons Lagrangian for $S L(2, R)$ :

$$
\begin{aligned}
& \mathcal{L}_{\mathrm{CS}}(A)=\kappa \varepsilon^{\rho \mu \nu}\left(A^{i}{ }_{\rho} \partial_{\mu} A_{i \nu}+\frac{1}{3} \varepsilon_{i j k} A^{i}{ }_{\rho} A^{j}{ }_{\mu} A^{k}{ }_{\nu}\right), \\
& D^{\mu}=-\kappa \varepsilon^{\mu \nu \rho} \bar{A}^{i}{ }_{\nu} A_{i \rho}=a \varepsilon^{\mu \nu \rho} b^{i}{ }_{\nu} \omega_{i \rho}, \quad \kappa \equiv \frac{a \ell}{2} .
\end{aligned}
$$

Now, we wish to clarify the meaning of the total derivative term appearing on the left hand side of (3.4a). Our asymptotic conditions (2.1) imply the following relations:

$$
A_{+}^{i}=\mathcal{O}_{1}, \quad \bar{A}_{-}^{i}=\mathcal{O}_{1} .
$$

It is well known that under such boundary conditions both Chern-Simons actions $I_{\mathrm{CS}}(A)$ and $I_{\mathrm{CS}}(\bar{A})$ are differentiable (see, e.g. Refs. $[12,25]$ ). Then, as a consequence of the identity (3.4a), we can define the improved gravitational action,

$$
\tilde{I}_{G}=I_{G}+\Delta, \quad \Delta \equiv a \int d^{3} x \partial_{\mu}\left(\varepsilon^{\mu \nu \rho} b^{i}{ }_{\nu} \omega_{i \rho}\right)
$$

which is also differentiable. Thus, the role of the boundary term $\Delta$ is to make the gravitational action differentiable under the variations which respect the boundary conditions (3.5). The identity (3.4a) can now be written in the simple form

$$
\tilde{I}_{G}=I_{\mathrm{CS}}(A)-I_{\mathrm{CS}}(\bar{A}),
$$

showing that 
the improved gravitational action in the teleparallel 3D gravity is equal to the difference of two Chern-Simons actions.

Thus, the Chern-Simons formulation of 3D gravity is possible not only in Riemannian GR [4], but also in the teleparallel gravity. The spacetime with torsion is an equally acceptable arena for formulating and discussing various features of boundary dynamics.

The result (3.7) implies that the classical central charge of the teleparallel theory has the value $c=12 \cdot 4 \pi \kappa=3 \ell / 2 G$, in agreement with Ref. [15].

\section{BOUNDARY DYNAMICS 2}

In this section we study the role of boundary conditions on the form of solutions of the teleparallel gravity in the Chern-Simons formulation, and identify the related boundary dynamics as the Liouville theory.

With the adopted conventions for the $S L(2, R)$ generators, the gauge potential $A^{i} \equiv$ $A^{i}{ }_{\mu} d x^{\mu}$ can be represented in the matrix form as

$$
T_{i} A^{i} \equiv A=\frac{1}{2}\left(\begin{array}{cc}
A^{1} & A^{+} \\
-A^{-} & -A^{1}
\end{array}\right),
$$

with the group indices written always as superscripts.

\section{A. Boundary conditions for $A_{\mu}^{i}$}

1. The asymptotic behavior of $b^{i}{ }_{\mu}$ and $\omega^{i}{ }_{\mu}$ implies the following conditions on the new variables $(3.2 \mathrm{a})$ :

$$
A=\frac{1}{2}\left(\begin{array}{cc}
\frac{d r}{r}+\mathcal{O}_{2} & \mathcal{O}_{1} \\
-\frac{2 r}{\ell} d x^{-}+\mathcal{O}_{1} & -\frac{d r}{r}+\mathcal{O}_{2}
\end{array}\right)
$$

In addition to this, we also have some more precise details about $A_{1}$ :

$$
A_{1}^{ \pm}=\mathcal{O}_{4}, \quad A_{1}^{1}=\frac{1}{r}+\mathcal{O}_{3} .
$$

2. The field equations (3.3) can be solved for $A$ as

$$
A=G^{-1} d G, \quad G \in S L(2, R) .
$$

If the spatial sections of spacetime contain no holes, this solution is valid also globally [5]. Since we are interested only in the asymptotic dynamics of gravity, we shall ignore the presence of holes.

Although $G$ is introduced as an arbitrary element of $S L(2, R)$, boundary conditions produce non-trivial restrictions on its form. To investigate these restrictions we note that, without loss of generality, the matrix $G$ can be represented as 


$$
G=g \rho, \quad \rho \equiv\left(\begin{array}{cc}
\sqrt{r / \ell} & 0 \\
0 & \sqrt{\ell / r}
\end{array}\right)
$$

where $g$ is also in $S L(2, R)$. Then, the relation

$$
A=G^{-1} d G=\rho^{-1} \mathcal{A} \rho+\rho^{-1} d \rho, \quad \mathcal{A} \equiv g^{-1} d g
$$

and the asymptotic conditions (4.1) for $A$, define the asymptotic behavior of $\mathcal{A}$ :

$$
\mathcal{A}=g^{-1} d g=\left(\begin{array}{cr}
0 & \mathcal{O}_{0} \\
-d x^{-} & 0
\end{array}\right)+\mathcal{O}_{2} .
$$

Taking into account the additional information (4.2) we find that $\mathcal{A}_{1}=\mathcal{O}_{3}$, which implies that the $\mathcal{O}_{0}$ term in $\mathcal{A}$ does not depend on $r$. Hence,

a) to leading order in $1 / r, \mathcal{A}$ depends only on $t$ and $\varphi: \mathcal{A}=\mathcal{A}(t, \varphi)$.

In other words, $\mathcal{A}$ is the field on the boundary. This result has a direct consequence on the form of $g$ (Appendix B):

b) to leading order in $1 / r$, the matrix $g$ depends only on $x^{-}$:

$$
g=g\left(x^{-}\right) .
$$

As a consequence, the $\mathcal{O}_{0}$ term in (4.5) must be a function of $x^{-}$, and we have

$$
\begin{aligned}
\text { i) } & \mathcal{A}_{+}=0, \\
\text { ii) } & \partial_{+} \mathcal{A}_{-}^{+}=0, \quad \mathcal{A}_{-}^{-}=2, \quad \mathcal{A}_{-}^{1}=0 .
\end{aligned}
$$

The higher order terms are not written explicitly, but are understood. Also, the term $\mathcal{A}_{1}$ has been dropped, as it trivially vanishes on the account of (4.6).

Thus, after the asymptotic conditions are taken into account, only one element of the original matrix $G$ in (4.3) is left to describe the boundary dynamics: $\mathcal{A}_{-}^{+}=\mathcal{A}_{-}^{+}\left(x^{-}\right)$.

\section{B. Boundary conditions for $\bar{A}_{\mu}^{i}$}

1. Applying the same procedure to the variable $\bar{A}$, we obtain

$$
\bar{A}=\frac{1}{2}\left(\begin{array}{cc}
-\frac{d r}{r}+\mathcal{O}_{2} & -\frac{2 r}{\ell} d x^{+}+\mathcal{O}_{1} \\
\mathcal{O}_{1} & \frac{d r}{r}+\mathcal{O}_{2}
\end{array}\right)
$$

and also

$$
\bar{A}_{1}^{ \pm}=\mathcal{O}_{4}, \quad \bar{A}_{1}^{1}=-\frac{1}{r}+\mathcal{O}_{3}
$$


2. The general solution for $\bar{A}$ is given as

$$
\bar{A}=\bar{G}^{-1} d \bar{G}, \quad \bar{G} \in S L(2, R) .
$$

In order to clarify the effect of boundary conditions on the form of $\bar{G}$, we write

$$
\bar{G}=\bar{g} \rho^{-1} .
$$

Then, the relation

$$
\bar{A}=\bar{G}^{-1} d \bar{G}=\rho \overline{\mathcal{A}} \rho^{-1}+\rho d \rho^{-1}, \quad \overline{\mathcal{A}}=\bar{g}^{-1} d \bar{g},
$$

and the asymptotic form (4.8) of $\bar{A}$, lead to

$$
\overline{\mathcal{A}}=\left(\begin{array}{cc}
0 & -d x^{+} \\
\mathcal{O}_{0} & 0
\end{array}\right)+\mathcal{O}_{2} .
$$

The additional conditions (4.9) imply that $\overline{\mathcal{A}}_{1}=\mathcal{O}_{3}$; hence,

a) to leading order in $1 / r, \overline{\mathcal{A}}$ depends only on $t$ and $\varphi: \overline{\mathcal{A}}=\overline{\mathcal{A}}(t, \varphi)$.

This result, in return, yields a precise information on the matrix $\bar{g}$ itself (Appendix B):

b) to leading order in $1 / r$, the matrix $\bar{g}$ depends only on $x^{+}$:

$$
\bar{g}=\bar{g}\left(x^{+}\right),
$$

Consequently, we have

$$
\begin{aligned}
\text { i) } & \overline{\mathcal{A}}_{-}=0, \\
\text { ii) } & \partial_{-} \overline{\mathcal{A}}_{+}^{-}=0, \quad \overline{\mathcal{A}}_{+}^{+}=-2, \quad \overline{\mathcal{A}}_{+}^{1}=0 .
\end{aligned}
$$

The only element of the matrix $\bar{G}$ in (4.10) that corresponds to a non-trivial boundary dynamics is $\overline{\mathcal{A}}_{+}^{-}=\overline{\mathcal{A}}_{+}^{-}\left(x^{+}\right)$.

We see that the whole content of our theory reduces to a 2-dimensional field theory at the boundary. The latter is described by the $S L(2, R)$ matrices $g\left(x^{-}\right)$and $\bar{g}\left(x^{+}\right)$, subject to the conditions (4.7) and (4.14). In what follows, this 2-dimensional theory will be analyzed in details.

\section{Liouville dynamics at the boundary}

The result of the previous analysis is completely expressed by Eqs. (4.7) and (4.14): after imposing the asymptotic conditions, there are only two components of the fields $A$ and $\bar{A}$ that remain non-trivial at the boundary: $\mathcal{A}_{-}^{+}\left(x^{-}\right)$and $\overline{\mathcal{A}}_{+}^{-}\left(x^{+}\right)$.

With a convenient parametrization, these two independent chiral fields can be associated to a single Liouville field $\phi=\phi\left(x^{-}, x^{+}\right)$. To show that, we introduce the new matrix $\gamma=g^{-1} \bar{g}$, and the related "currents" $K=\gamma^{-1} d \gamma$ and $\bar{K}=\gamma d \gamma^{-1}$. Then we find 


$$
\begin{aligned}
& K_{+}=\gamma^{-1} \partial_{+} \gamma=\bar{g}^{-1} \partial_{+} \bar{g}=\overline{\mathcal{A}}_{+}, \\
& \bar{K}_{-}=\gamma \partial_{-} \gamma^{-1}=g^{-1} \partial_{-} g=\mathcal{A}_{-},
\end{aligned}
$$

and the Eqs. (4.7) and (4.14) lead to

$$
\begin{array}{ll}
\partial_{-} K_{+}=0, & \partial_{+} \bar{K}_{-}=0, \\
K_{+}^{+}=-2, & \bar{K}_{-}^{-}=2, \\
K_{+}^{1}=0, & \bar{K}_{-}^{1}=0 .
\end{array}
$$

In return, the conditions (4.16) imply (4.7) and (4.14) up to a constant $S L(2, R)$ matrix. This means that the complete content of the equations (4.7) and (4.14) is basically expressed in terms of $K$ and $\bar{K}$.

For arbitrary $\gamma$, the boundary conditions $\partial_{-} K_{+}=0$ represent the set of three field equations for the Wess-Zumino-Novikov-Witten (WZNW) model; they are equivalent to $\partial_{+} \bar{K}_{-}=0$. After introducing the Gauss coordinates $(X, \phi, Y)$ to parametrize the $S L(2, R)$ group manifold, these equations, combined with $(4.16 \mathrm{~b})$, reduce to the Liouville equation (Appendix C),

$$
\partial_{-} \partial_{+} \phi-2 e^{\phi}=0,
$$

while the remaining two conditions (4.16c) yield:

$$
2 X=-\partial_{-} \phi, \quad 2 Y=\partial_{+} \phi .
$$

The general solution for the Liouville field is given in terms of two chiral functions, hence we have the correct balance of the dynamical degrees of freedom at the boundary. Therefore,

the boundary dynamics of the teleparallel gravity can be identified with Liouville theory.

\section{Energy-momentum tensor}

The non-trivial fields at the boundary, $\overline{\mathcal{A}}_{+}^{-}$and $\mathcal{A}_{-}^{+}$, can be expressed in terms of the Liouville field as follows:

$$
\begin{aligned}
& \overline{\mathcal{A}}_{+}^{-}=K_{+}^{-}=\frac{1}{2}\left(\partial_{+} \phi\right)^{2}-\partial_{+}^{2} \phi=\theta_{++}, \\
& \mathcal{A}_{-}^{+}=\bar{K}_{-}^{+}=-\frac{1}{2}\left(\partial_{-} \phi\right)^{2}+\partial_{-}^{2} \phi=-\theta_{--},
\end{aligned}
$$

where $\theta_{\mp \mp}$ are components of the Liouville energy-momentum tensor. Then, we can return to the original gravitational variables discussed in section II,

$$
\mathcal{A}_{-}^{+}=\hat{\Omega}_{-}^{+}=\frac{2}{\ell} \hat{B}_{-}^{+}, \quad \overline{\mathcal{A}}_{+}^{-}=\hat{\Omega}_{+}^{-}-\frac{2}{\ell} \hat{B}_{+}^{-}=-\frac{2}{\ell} \hat{B}_{+}^{-},
$$

and derive the relations

$$
\theta_{++}=-\frac{2}{\ell} \hat{B}_{+}^{-}, \quad \theta_{--}=-\frac{2}{\ell} \hat{B}_{-}^{+} .
$$


Using the known symmetry of the Liouville theory, $\delta_{0} \phi=-\varepsilon \cdot \partial \phi-\partial \cdot \varepsilon$, with $\varepsilon=\left(T^{+}, T^{-}\right)$, one finds the transformation law

$$
\begin{aligned}
& \delta_{0} \theta_{++}=-T^{+} \partial_{+} \theta_{++}-2 \partial_{+} T^{+} \theta_{++}+\partial_{+}^{3} T^{+}, \\
& \delta_{0} \theta_{--}=-T^{-} \partial_{-} \theta_{--}-2 \partial_{-} T^{-} \theta_{--}+\partial_{-}^{3} T^{-},
\end{aligned}
$$

in agreement with (2.7). Thus,

the boundary fields $\hat{B}_{+}^{-}\left(x^{+}\right)$and $\hat{B}_{-}^{+}\left(x^{-}\right)$are proportional to the $(++)$and $(--)$ components of the Liouville energy-momentum tensor.

This agrees with the result of Ref. [8], which is, however, based on purely geometric arguments.

\section{CONCLUDING REMARKS}

In the present paper we analyzed the nature of asymptotic dynamics in the teleparallel 3D gravity, starting from the asymptotic AdS configuration (2.1) for the triad $b^{i}{ }_{\mu}$ and the connection $\omega^{i}{ }_{\mu}$. The full set of gauge symmetries is naturally split into two parts: the conformal symmetry and the proper gauge symmetry [15].

Using the asymptotic field equations, we found that there are only two non-trivial dynamical modes at the boundary: $\hat{B}_{-}^{+}\left(x^{-}\right)$and $\hat{B}_{+}^{-}\left(x^{+}\right)$. Their transformation laws show that the boundary dynamics is described as a conformal field theory with the classical central charge $c=3 \ell / 2 G$.

An important step in clarifying the structure of the boundary field theory is made by showing that the improved action of the teleparallel 3D gravity is given as a difference of two Chern-Simons actions. This result leads us to recognize Liouville theory as the dynamical structure at the boundary. The fact that the same result is found also in Riemannian 3D gravity [4-11] indicates very strongly that the nature of boundary dynamics does not depend on the geometric context, but only on the boundary conditions.

\section{ACKNOWLEDGMENTS}

One of us (M.B.) would like to thank Friedrich Hehl for reading the manuscript. This work was partially supported by the Serbian Science foundation, Serbia. 


\section{APPENDIX A: PROPER GAUGE TRANSFORMATIONS}

Using the notation $\hat{\delta} \equiv \delta_{0}(T=S=0)$ for the proper gauge variation, the general transformation law (1.1a) leads to the following asymptotic relations:

$$
\begin{array}{ll}
\hat{\delta} \hat{B}_{-}^{+}=\hat{\delta} \hat{B}_{+}^{-}=0, & \hat{\delta} \hat{\Omega}_{-}^{+}=\hat{\delta} \hat{\Omega}_{+}^{-}=0, \\
\hat{\delta} \hat{B}_{+}^{+}=\ell \hat{\theta}-2 \hat{\xi}^{1}, & \hat{\delta} \hat{\Omega}_{+}^{+}=0, \\
\hat{\delta} \hat{B}_{-}^{-}=-\ell \hat{\theta}, & \hat{\delta} \hat{\Omega}_{-}^{-}=-2 \hat{\theta}, \\
\hat{\delta} \hat{B}_{+}^{1}=-\partial_{+} \hat{\xi}^{1}+\frac{\ell}{2} \hat{\theta}^{-}, & \hat{\delta} \hat{\Omega}_{+}^{1}=-\partial_{+} \hat{\theta}, \\
\hat{\delta} \hat{B}_{-}^{1}=-\partial_{-} \hat{\xi}^{1}-\frac{\ell}{2} \hat{\theta}^{+}, & \hat{\delta} \hat{\Omega}_{-}^{1}=-\partial_{-} \hat{\theta}-\hat{\theta}^{+}, \\
\hat{\delta} \hat{B}_{1}^{+}=4 \hat{\xi}^{+}-\hat{\theta}^{+}, & \hat{\delta} \hat{\Omega}_{1}^{+}=\frac{2}{\ell} \hat{\theta}^{+}, \\
\hat{\delta} \hat{B}_{1}^{-}=4 \hat{\xi}^{-}+\hat{\theta}^{-}, & \hat{\delta} \hat{\Omega}_{1}^{-}=\frac{4}{\ell}\left(\hat{\theta}^{-}+2 \hat{\xi}^{-}\right), \\
\hat{\delta} \hat{B}_{1}^{1}=\frac{2}{\ell} \hat{\xi}^{1}, & \hat{\delta} \hat{\Omega}_{1}^{1}=\frac{2}{\ell} \hat{\theta},
\end{array}
$$

where $\hat{\theta} \equiv \hat{\theta}^{1}+\hat{\xi}^{1} / \ell$. The equality in the above expressions is the equality up to $\mathcal{O}_{1}$ terms (which are omitted for simplicity). The last six equations show that the proper gauge freedom can be fixed as in (2.4).

\section{APPENDIX B: CONDITIONS (4.6) AND (4.13)}

The general expression (4.5) for $\mathcal{A}=\mathcal{A}(t, \varphi)$ can be written as follows:

$$
\begin{aligned}
& \mathcal{A}_{+}=g^{-1} \partial_{+} g=\left(\begin{array}{cc}
0 & \partial_{+} \alpha \\
0 & 0
\end{array}\right), \\
& \mathcal{A}_{-}=g^{-1} \partial_{-} g=\left(\begin{array}{cc}
0 & \beta \\
-1 & 0
\end{array}\right),
\end{aligned}
$$

where $\alpha$ and $\beta$ are functions of $t$ and $\varphi$. The first condition implies

$$
g=U\left(\begin{array}{ll}
1 & \alpha \\
0 & 1
\end{array}\right), \quad U=U\left(x^{-}\right)
$$

Substituting this expression into the second condition, one finds

$$
U^{-1} \partial_{-} U=\left(\begin{array}{cc}
-\alpha & \beta+\alpha^{2}-\partial_{-} \alpha \\
-1 & \alpha
\end{array}\right) .
$$

Consequently, $\alpha=\alpha\left(x^{-}\right), \beta=\beta\left(x^{-}\right)$, which proves (4.6).

Similarly, the expression (4.12) for $\overline{\mathcal{A}}=\overline{\mathcal{A}}(t, \varphi)$ yields 


$$
\begin{aligned}
& \overline{\mathcal{A}}_{-}=\bar{g}^{-1} \partial_{-} \bar{g}=\left(\begin{array}{cc}
0 & 0 \\
\partial_{-} \bar{\alpha} & 0
\end{array}\right), \\
& \overline{\mathcal{A}}_{+}=\bar{g}^{-1} \partial_{+} \bar{g}=\left(\begin{array}{cc}
0 & -1 \\
\bar{\beta} & 0
\end{array}\right),
\end{aligned}
$$

with $\bar{\alpha}=\bar{\alpha}(t, \varphi), \bar{\beta}=\bar{\beta}(t, \varphi)$. The first condition implies

$$
\bar{g}=\bar{U}\left(\begin{array}{cc}
1 & 0 \\
\alpha & 1
\end{array}\right), \quad \bar{U}=\bar{U}\left(x^{+}\right) .
$$

Then, the substitution of this expression into the second condition leads to $\bar{\alpha}=\bar{\alpha}\left(x^{+}\right)$, $\bar{\beta}=\bar{\beta}\left(x^{+}\right)$, which proves $(4.13)$.

\section{APPENDIX C: FROM WZNW TO LIOUVILLE}

In this appendix we recall the process of reduction of the WZNW field equations for the group $S L(2, R)$, to Liouville equation.

Every element $\gamma$ of $S L(2, R)$, in a neighborhood of identity, admits the Gauss decomposition:

$$
\gamma=\left(\begin{array}{cc}
1 & X \\
0 & 1
\end{array}\right)\left(\begin{array}{cc}
e^{\phi / 2} & 0 \\
0 & e^{-\phi / 2}
\end{array}\right)\left(\begin{array}{cc}
1 & 0 \\
Y & 1
\end{array}\right),
$$

where $(X, \phi, Y)$ are group coordinates. With the above expression for $\gamma$, the Lie algebra valued 1-form $K=\gamma^{-1} d \gamma=T_{i} K^{i}$, defines the quantity $K^{i}$, the vielbein on the group manifold:

$$
\begin{aligned}
& K^{+}=2 e^{-\phi} d X \\
& K^{1}=2 Y e^{-\phi} d X+d \phi \\
& K^{-}=-2\left(-Y^{2} e^{-\phi} d X-Y d \phi+d Y\right) .
\end{aligned}
$$

Similarly, $\bar{K}=\gamma d \gamma^{-1}=T_{i} \bar{K}^{i}$ defines $\bar{K}^{i}$ :

$$
\begin{aligned}
& \bar{K}^{+}=2\left(-d X+X d \phi+X^{2} e^{-\phi} d Y\right), \\
& \bar{K}^{1}=-d \phi-2 X e^{-\phi} d Y \\
& \bar{K}^{-}=2 e^{-\phi} d Y .
\end{aligned}
$$

The relations $\partial_{-} K_{+}^{i}=0$ are equivalent to $\partial_{+} \bar{K}_{-}^{i}=0$, and produce the field equations of the WZNW model:

$$
\begin{aligned}
& \partial_{-}\left(e^{-\phi} \partial_{+} X\right)=0, \\
& \partial_{-} \partial_{+} \phi+2 \partial_{-} Y \partial_{+} X e^{-\phi}=0, \\
& \partial_{+}\left(e^{-\phi} \partial_{-} Y\right)=0 .
\end{aligned}
$$

Under the boundary conditions (4.16b), the second of these equations is easily seen to reduce to the Liouville equation (4.17a). 


\section{REFERENCES}

[1] S. Deser, R. Jackiw and G 't Hooft, Three-dimensional Einstein gravity: dynamics of flat space, Ann. Phys. (N.Y) 152, 220 (1984); S. Deser and R. Jackiw, Threedimensional cosmological gravity: dynamics of constant curvature, Ann. Phys. (N.Y) 153, 405 (1984).

[2] See also the reviews: S. Carlip, Lectures on (2+1)-dimensional gravity, preprint grqc/9503024; S. Carlip, Quantum Gravity in 2+1 Dimensions (Cambridge University Press, Cambridge, 1998).

[3] J. D. Brown and M. Henneaux, Central charges in the canonical realization of asymptotic symmetries: an example from three-dimensional gravity, Commun. Math. Phys. 104, 207 (1986); see also M. Henneaux, Energy-momentum, angular momentum, and superscharge in 2+1 dimensions, Phys. Rev. D29, 2766 (1984); J. D. Brown, Lower dimensional gravity (Singapore, World Scientific, 1988).

[4] E. Witten, 2+1 dimensional gravity as an exactly soluble system, Nucl. Phys. B311, 46 (1988); A. Achucarro and P. Townsend, A Chern-Simons action for three-dimensional anti-de Sitter supergravity theories, Phys. Lett. B180, 89 (1986).

[5] O. Coussaert, M. Henneaux and P. van Driel, The asymptotic dynamics of threedimensional Einstein gravity with negative cosmological constant, Class. Quantum Grav. 12, 2961 (1995).

[6] M. Bañados, Global charges in Chern-Simons theory and $2+1$ black hole, Phys. Rev. D52, 5861 (1996).

[7] A. Strominger, Black hole entropy from near-horizon microstates, J. High Energy Phys. 9802, 009 (1998).

[8] J. Navaro-Salas and P. Navaro, A Note on Einstein gravity on $\mathrm{AdS}_{3}$ and boundary conformal field theory, Phys. Lett. B439, 262 (1998).

[9] M. Bañados, Three-dimensional quantum geometry and black holes, Invited talk at the Second Meeting "Trends in Theoretical Physics", held in Buenos Aires, December, 1998, preprint hep-th/9901148.

[10] M. Bañados, Notes on black holes and three-dimensional gravity, Proceedings of the VIII Mexican School on Particles and Fields, AIP Conf. Proc. 490, 198 (1999).

[11] M. Bañados, T. Brotz and M. Ortiz, Boundary dynamics and the statistical mechanics of the $2+1$ dimensional black hole, Nucl. Phys. B545, 340 (1999).

[12] J. Zanelli, Chern-Simons gravity: from $2+1$ to $2 \mathrm{n}+1$ dimensions, Lectures at the XX Econtro de Fisica de Particulas e Campos, Brasil, October 1998, and at the Fifth La Hechicera School, Venezuela, November 1999, Braz. J. Phys. 30, 251 (1999).

[13] F. W. Hehl, Four lectures in Poincaré gauge theory, in Proceedings of the 6th Course of the School of Cosmology and Gravitation on Spin, Torsion, Rotation and Supergravity, held at Erice, Italy, 1979, eds. P. G. Bergmann, V. de Sabbata (Plenum, New York, 1980) p. 5; E. W. Mielke, Geometrodynemics of Gauge Fields - On the Geometry of Yang-Mills and Gravitational Gauge Theories (Academie-Verlag, Berlin,1987).

[14] M. Blagojević, Gravitation and Gauge Symmetries (Bristol, Institute of Physics Publishing, 2002); M. Blagojević, Three lectures on Poincaré gauge theory, preprint gr-qc/0302.

[15] M. Blagojević and M. Vasilić, Asymptotic symmetries in 3D gravity with torsion, Phys. Rev. D67, 084032 (2003). 
[16] E. W. Mielke, P. Baekler, Topological gauge model of gravity with torsion, Phys. Lett. A156, 399 (1991).

[17] P. Baekler, E. W. Mielke, F. W. Hehl, Dynamical symmetries in topological 3D gravity with torsion, Nuovo Cim. B107, 91 (1992).

[18] A. García, F. W. Hehl, C. Heinecke and A. Macías, Exact vacuum solution of a (1+2)dimensional Poincaré gauge theory: BTZ solution with torsion, Phys. Rev. D67, 124016 (2003).

[19] C. Møller, On the crisis in the theory of gravitation and a possible solution, Mat. Fys. Scr. Dan. Vid. Selsk. 89, No. 13 (1978); K. Hayashi and T. Shirafuji, New general relativity, Phys. Rev. D19, 3524 (1979).

[20] J. Nitsch, The macroscopic limit of the Poincaré gauge field theory of gravitation, in Cosmology and Gravitation: Spin, torsion, Rotation and Supergravity, eds. P. G. Bergmann and V. de Sabbata (Plenum, New York, 1980) p. 63; F. W. Hehl, J. Nitsch and P. von der Heyde, Gravitation and the Poincaré gauge field theory with quadratic Lagrangian, in General Relativity and Gravitation - One Hundred Years After the Birth of Albert Einstein, ed. A. Held (Plenum, New York, 1980) vol. 1, p. 329.

[21] T. Kawai, Teleparallel theory of (2+1)-dimensional gravity, Phys. Rev. D48, 5668 (1993); Poincaré gauge theory of (2+1)-dimensional gravity, Phys. Rev. D49, 2862 (1994); Exotic black hole solution in teleparallel theory of (2+1)-dimensional gravity, Prog. Theor. Phys. 94, 1169 (1995); A. A. Sousa, J. W. Maluf, Canonical formulation of gravitational teleparallelism in $2+1$ dimensions in Schwinger's time gauge, Prog. Theor. Phys. 104, 531 (2000).

[22] S. W. Hawking and G. F. R. Ellis, The Large Scale Structure of Spacetime (Cambridge, Cambridge University Press, 1973).

[23] M. Henneaux and C. Teitelboim, Asymptotically anti-de Sitter spaces, Commun. Math. Phys. 98, 391 (1985).

[24] S. Elitzur, G. Moore and N. Seiberg, Remarks on the canonical quantization of the Chern-Simons-Witten theory, Nucl. Phys. B326, 108 (1989); M. Bañados and A. Gomberoff, Black hole entropy of the Chern-Simons formulations of $2+1$ gravity, Phys. Rev. D55, 6162 (1997);

[25] M. Bañados, C. Teitelboim and J. Zanelli, The black hole in three-dimensional spacetime, Phys. Rev. Lett. 16, 1849 (1993); M. Bañados, M. Henneaux, C. Teitelboim and J. Zanelli, Geometry of 2+1 black hole, Phys. Rev. D48, 1506 (1993). 